\title{
Effect of Haematogenous Oxidation Therapy with Ul- traviolet-C Irridation in Alloxan-Induced Diabetes and Poloxamer 407-Induced Hyperlipidemia in Rabbits
}

\author{
Gareeballah Osman Adam ${ }^{1,2}$, Kyung-Hun Lee', JongsukKim¹, Byung-Yong Park ${ }^{3}$, Jin-Shang Kim ${ }^{1}$, \\ SeungilKim ${ }^{4}$, Yeong-Seok Yang ${ }^{5}$, Hyung-Sub Kang ${ }^{*}$, Gi-BeumKim ${ }^{*}$ \\ ${ }^{1}$ Department of Pharmacology, College of Veterinary Medicine, Chonbuk National University, Iksan Campus, 79 Gobong-ro, Iksan-si, Jeollabuk-do 54596Republic \\ of Korea \\ ${ }^{2}$ Department of Veterinary Medicine and Surgery, College of Veterinary Medicine, Sudan University of Science and Technology, P.O. Box No. 204, Hilat Kuku, \\ Khartoum, Sudan \\ ${ }^{3}$ Department of Veterinary Anatomy, College of Veterinary Medicine, Chonbuk National University, Iksan Campus, 79 Gobong-ro, Iksan-si, Jeollabuk-do 54596 \\ Republic of Korea \\ ${ }^{4}$ Filntox Clinic, 555 Baeje-daero, Deokjin-gu, Jeonju-si, Jeollabuk-do, 54898 Republic of Korea \\ ${ }^{5}$ Division of Pharmaceutical Engineering, Woosuk University, Samnye-ro, Samnye-eup, Wanju 565-701, Republic of Korea \\ ${ }^{6}$ Korea Pickle Co., LTD., Sunchang-eup, Sunchang-gun, Jeollabuk-do, 56048 Republic of Korea
}

*Corresponding author: Hyung-Sub Kang, Department of Pharmacology, College of Veterinary Medicine, Chonbuk National University, Iksan Campus, 79 Gobong-ro, Iksan-si, Jeollabuk-do 54596Republic of Korea, E-mail: kang-hs@chonbuk.ac.kr

Gi-Beum Kim, Korea Pickle Co., LTD., Sunchang-eup, Sunchang-gun, Jeollabuk-do, 56048 Republic of Korea, E-mail: kgb70@jbnu.ac.kr

\begin{abstract}
Background: Recently diabetes and hyperlipidemia (HL) considered a major source of mortality. Chemical treatments could minimize the symptoms but, still the disease exists. Previously Ultraviolet was used for treatment of ailments related to infection and metabolism.

Methods: The study evaluates the effects of Haematogenous Oxidation Therapy (HOT) on the blood when a low dose of Ultraviolet-C (UV-C) is directly irradiated to the blood in a diabetic rabbit model and to evaluate the effects of treatment on diabetic rabbit. Type 1 Diabetes and hyperlipidemia were induced by intravenous injection of alloxan monohydrate and subcutaneous injection of poloxamer 407, respectively. A $10 \mathrm{ml}$ blood was collected from diabetic rabbits, blood was being perfused with oxygen for 10 seconds and UV-C was irradiated to the blood, UV-irradiated blood was transfused back to the original rabbits. The HOT treatment was performed a total of 10 times. It was evaluated the effects of the HOT treatment on diabetes and HL through hematological and biochemical analyses before and after HOT treatment were performed.

Results: The results indicated that the reduced body weight was increased and blood glucose levels were significantly reduced after the HOT treatment was performed when compared to those prior to the HOT treatment. In addition, CRE, BUN and UA levels indicating renal functions were significantly reduced when compared to those prior to the HOT treatment. When the HOT treatment was performed in a diabetic and HL rabbit model, our results indicate that blood glucose levels and lipids profile were improved.

Conclusions: Biochemical and Hematological analyst was demonstrating that the HOT was effective to alleviate diabetes and HL.
\end{abstract}

Keywords: Haematogenous oxidation therapy; Insulin; Type 1 diabetes rabbit model; Hyperlipidemia; poloxamer; Total cholesterol; Creatinine and Poloxamer 407-Induced Hyperlipidemia in Rabbits. (2018) J diab Obes 5(1): 48- 54.

Copyright: (C) 2018 Kang, H.S., Gi-Beum, K. This is an Open access article distributed under the terms of Creative Commons Attribution 4.0 International License. 


\section{Background}

Ultra violet irradiation (UBI) of blood was hailed as a miracle therapy for treating serious infections in the mid of 19th century. However, this time period coincided with the widespread introduction of penicillin antibiotics, which were rapidly found to be an even bigger miracle therapy. Moreover, another major success of UBI, which was becoming used to treat polio, was also eclipsed by the introduction of the Salk vaccine. Starting in the 1960s UBI fell into disuse in the West and has now been called "the cure that time forgot"[1].

Recently, there has been a gradual increase in mortality due to coronary heart diseases (CHDs). Factors such as hypercholesterolemia, cigarette smoking, diabetes mellitus, and sedentary lifestyle are key contributors to the development of hyperlipidemia (HL) and atherosclerotic cardiovascular disease. Apart from these factors, there are evidences that suggest certain chemicals can also cause hyperlipidemia and may substantially lead to CHDs. Poloxamer 407 was one such example ${ }^{[2]}$.

Poloxamer 407-induced hypertriglyceridemia and hypercholesterolemia are well known incident. Elevated triglycerides and inhibition due to lipoprotein lipase was the main feature of poloxamar 407- induced hyperlipidemia ${ }^{[3]}$.

Diabetes was a metabolic disease with high blood glucose levels caused by the metabolic disorder resulting from defects of hormones such as insulin, glucagon or glucocorticoids involved in the metabolism of glucose or by abnormal reactions in the pathway ${ }^{[4]}$. Because the diabetes was characterized by high blood glucose levels, which leads to a wide range of malfunctions in the metabolic control over carbohydrates, proteins, fats, and electrolytes, it was closely associated with increases in various chronic degenerative diseases ${ }^{[5]}$. Diabetes emerges as the disease causing social problems because it has a very high prevalence as a typical chronic metabolic disease. Diabetes goes beyond endemic limits and was becoming an epidemic internationally ${ }^{[6,7]}$. The incidence of diabetes was very low in Korea when compared to that in Western countries. However, the incidence was gradually increasing in Korea due to recent changes in diet, environment, and lifestyle ${ }^{[8,9]}$.

Oral hypoglycemic agents and insulin formulation for the treatment for the diabetes are continuously developed. However, because it was the chronic disease which was not fully cured once patients develop diabetes, it causes a serious prob$\operatorname{lem}^{[10]}$.

Thus, in this study, it was evaluated the effects of Haematogenous Oxidation Therapy (HOT) blood irradiation on the diabetes and HL using physical methods with UV light rather than drug therapy such as insulin and statins injection in order to get over diabetes- and HL-causing serious problems with an emphasis on diabetes.

\section{Methods}

\section{Design of ultraviolet blood irradiation device}

In this study, HOT device was produced to identify effects of ultraviolet blood irradiation on the blood in a diabetic animal model. Figure 1 shows a simple drawing and photo of the HOT device. In brief, a fixing holder for quartz cuvette was installed in the center and ultraviolet (UV) lamps were installed on both sides of the holder. The UV lamps are designed to adjust the distance $(5 \mathrm{~mm} \sim 120 \mathrm{~mm}$ ) from the holder in the center. Ultraviolet blood irradiation was performed in the cuvette. Vacuum bottle

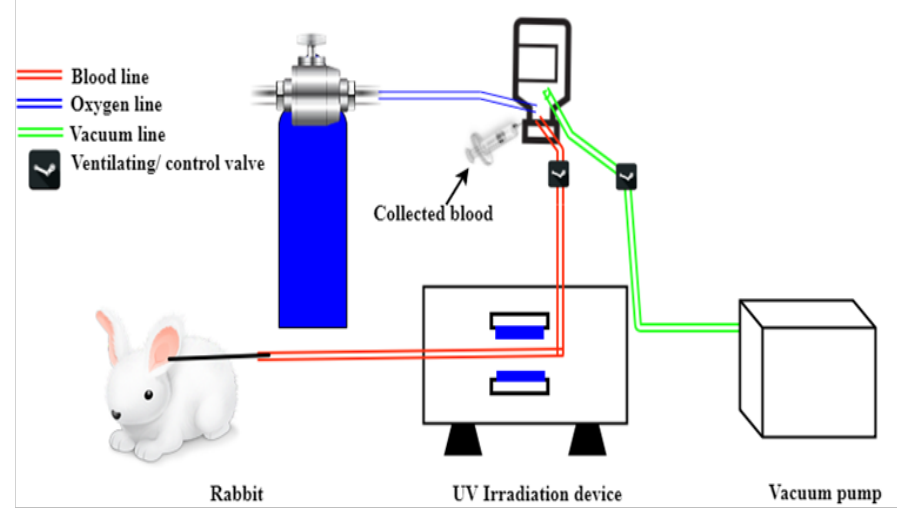

Figure 1: Schematic illustration and photographic of the HOT device.

\section{Experimental animals}

Adult male eight New Zealand White rabbits of body weighing $2-2.5 \mathrm{~kg}$ were used in the study. All the rabbits were kept in cages with wide square mesh at the bottom to avoid coprophagy and maintained under controlled conditions of humidity, temperature $\left(22 \pm 2^{\circ} \mathrm{C}\right)$ and 12 hours' light and dark cycle. Food and water were provided ad libitum. They were fasted for 18 hours prior to the experiment, allowing free access to water only. The experimental protocols were approved by the Institutional Animal Ethics Committee. All experimental protocols (CBU20130010) were approved by the Committee on the Care of Laboratory Animal Resources, Chonbuk National University and were conducted in accordance with the Guide for the Care and Use of Laboratory.

\section{Induction of experimental diabetes - Procedure for injecting alloxan monohydrate}

The 10 rabbits weighing between 2 to $2.5 \mathrm{~kg}$ were made diabetic by injecting intravenously $110 \mathrm{mg} / \mathrm{kg}$ body weight of alloxan monohydrate (A7413, Aldrich), a dose confirmed to induce diabetes by our previous study ${ }^{[1]}$. Before giving alloxan, the normal blood glucose levels of all rabbits were estimated. After 2 hours of alloxan injection, the 5\% Dextrose injected to the all-diabetic rabbits intraperitoneally to prevent a hypoglycemic condition of rabbits with alloxan. After 72 hours of alloxan injection, the blood glucose levels of all surviving rabbits were determined by the glucose oxidase method.

\section{Induction of experimental hyperlipidemia - Procedure for injecting poloxamar 407}

Poloxamar 407 (Sigma) was prepared at a concentration of $30 \%$ $(\mathrm{w} / \mathrm{w})$ in sterile distilled water. The 10 rabbits weighing between 2 to $2.5 \mathrm{~kg}$ were made hyperlipidemic by injecting $137.5 \mathrm{mg} / \mathrm{kg}$ $(0.7 \mathrm{ml} / \mathrm{kg})$ subcutaneously after shaving the dorsal surface of injection, dose repeated weekly for 10 weeks.

\section{Haematogenous Oxidation Therapy}

It was confirmed that the diabetes was induced by measuring blood glucose levels in rabbits at 72 hours (3 days) after alloxan was injected. The blood was collected from diabetic rabbits after 
1 week. The blood was perfused with oxygen for 10 seconds. For the HOT, UV was irradiated to the blood collected through auto transfusion and the blood was transfused back to the original rabbit. Anticoagulation Sodium Citrate Solution (BOIN ACDA SOLN, SBD Co., Ltd.) was used to prevent coagulation of the blood when it was collected. $10 \mathrm{ml}$ blood was collected from the vein by using $20 \mathrm{ml}$ syringe (needle gauge 26). UV light with the intensity of $10.290 \mathrm{~J} / \mathrm{cm}^{2}$ was irradiated to the blood which passes at a constant flow rate using the syringe pump in the HOT device. Once UV was irradiated to the blood, the blood collected on the syringe in the other side of the cuvette. After the HOT was performed, the blood transfused back to the original rabbit. The HOT treatment was performed a total of 10 times. Rabbits were stably raised in a laboratory animal breeding facility. Food and water were sufficiently supplied.

\section{Biochemical analysis}

Blood was collected from the ear marginal vein. A Nova Stat Profile $^{\circledR} \mathrm{pHOx}^{\circledR}$ Ultraanalyzer (NOVA Biomedical Corp., Waltham, MA, USA) was used to measure blood gas, electrolytes, and anion gap. After clotting, blood serum was separated by centrifugation at $3000 \mathrm{rpm}$ for $20 \mathrm{~min}$. The levels of glucose, enzymes, lipids, and proteins were analyzed using a Model 7020 auto analyzer (Hitachi, Tokyo, Japan).

\section{Serum insulin and HbAlc measurements}

Fasting serum insulin levels were measured with a rat insulin enzyme-linked immune absorbent assay kit (Life Span Bio Sciences, Inc., LS-F21890) according to the manufacturer's protocol. HbA1c ELWASA kit (ALPCO Diagnostics, Windham, NH, USA) were quantities using commercially available kits according to the manufacturers' protocols.

\section{Statistical analysis}

Data were expressed as means \pm standard errors of the mean (SEMs). Differences between groups were evaluated by oneway analysis of variance with the Bonferroni post hoc test or by calculation of Spearman's rank correlation coefficient, as appropriate, using Prism 5.03 (GraphPad Software Inc., San Diego, CA, USA). Statistical significance was set at $\mathrm{p}<0.05$.

\section{Results}

\section{Serum glucose, insulin, and HbA1c concentration}

In the diabetic rabbit model induced by alloxan, glucose was increased significantly compared to normal rabbits as shown in figure 2. However, in the diabetic rabbit model undergoing the HOT treatments, glucose levels were reduced over the course of the treatment.
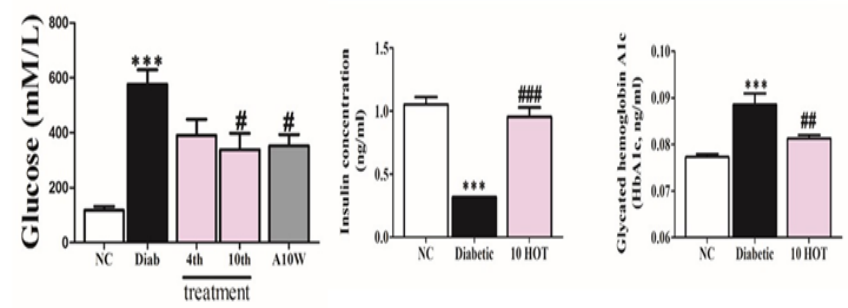

Figure 2: Effects of HOT on glucose, insulin, and glycated hemoglobin A1c (HbA1c) concentration of serum in alloxan-induced diabetics rab- bits. Data were reported as means \pm SEMs $(\mathrm{n}=10){ }^{*}: \mathrm{p}<0.05 ; * *$ : $\mathrm{p}$ $<0.01$; and $* * *: \mathrm{p}<0.001$, Bonferroni post hoc test following oneway ANOVA versus the NC; normal control prior diabetes was induced and before HOT: $p<0.05$; \#\#: $\mathrm{p}<0.01$; and \#\#\#: $\mathrm{p}<0.001$, Bonferroni post hoc test following one-way ANOVA versus Diab; diabetic (after diabetes induced and prior HOT),4th, 10th treatment, and A10W; fourth treatment, tenth treatment, and 10 weeks after last treatment respectively.

Insulin concentration in the blood was significantly decreased in the diabetic group without HOT treatment compared to the normal group (NC) as shown in figure 3. However, the insulin concentration in the HOT group was almost similar to that of NC. Therefore, an increase in the insulin concentration of blood in diabetic groups receiving the HOT treatment reduced blood glucose. The HbA1c in the diabetic group without HOT treatment was significantly increased compared to the normal group. However, the $\mathrm{HbA} 1 \mathrm{c}$ in the diabetic group that received the HOT treatment was significantly reduced compared to the group without the HOT treatment.
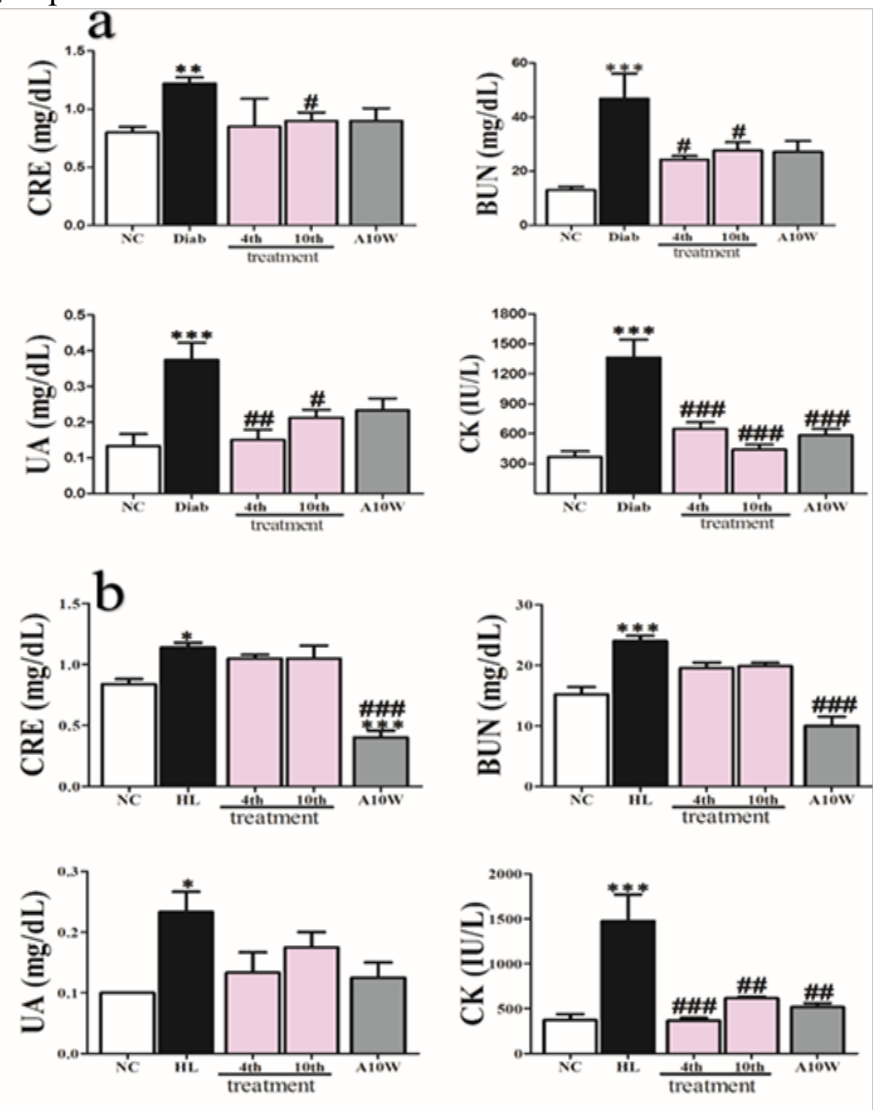

Figure 3: Effects of HOT on the renal characteristics by serum analysis in alloxan-induced diabetes (a) and 407-induced hyperlipidemic(b) rabbits.CRE, creatinine; BUN, blood urea nitrogen; UA, uric acid; creatine kinase $(\mathrm{CK})$. Data were reported as means \pm SEMs $(\mathrm{n}=10)$. *: $\mathrm{p}<$ $0.05 ; * *: \mathrm{p}<0.01$; and $* * *: \mathrm{p}<0.001$, Bonferroni post hoc test following oneway ANOVA versus the NC; normal control prior diabetes was induced and before HOT: $\mathrm{p}<0.05$; \#\#: $\mathrm{p}<0.01$; and \#\#: $\mathrm{p}<0.001$, Bonferroni post hoc test following one-way ANOVA versus Diab; diabetic (after diabetes induced and prior HOT), 4th, 10th treatment, and A10W; fourth treatment, tenth treatment, and 10 weeks after last treatment respectively.

\section{Kidney function test}

In diabetes and HL rabbit prior HOT treatment; CRE, BUN, UA 
and CK levels were elevated significantly when compared to normal group as shown in figure $4 \mathrm{a}$ and $4 \mathrm{~b}$. However, after HOT treatments sessions were started these values were significantly reduced when compared to those before HOT was applied.
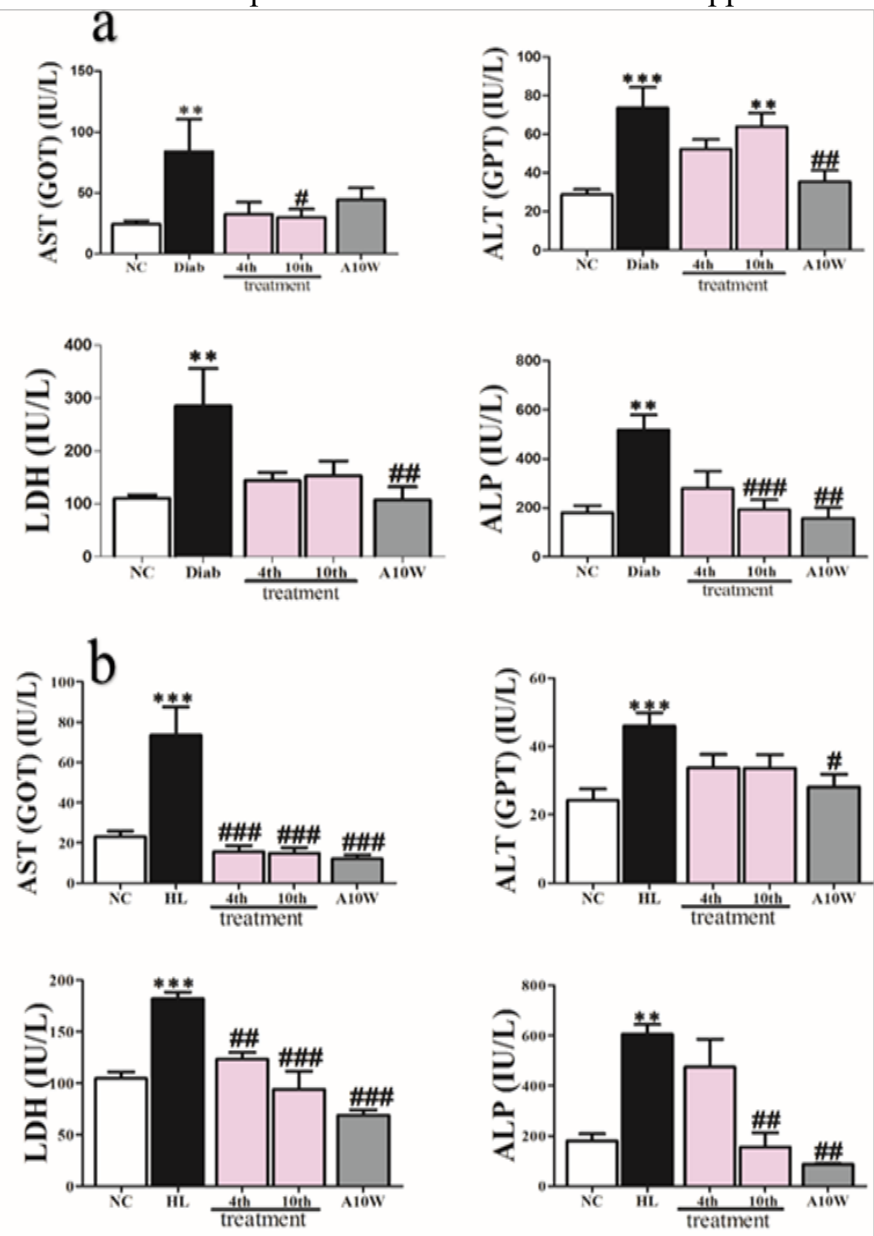

Figure 4: Effect of HOT on the liver characteristic by serum metabolic enzymes analysis in alloxan-induced diabetic (a) and 407-induced hyperlipidemic(b) rabbits. AST, aspartate aminotransferase; ALT, alanine aminotransaminase; LDH, lactate dehydrogenase; ALP, alkaline phosphatase. Data were reported as means \pm SEMs $(n=10) . *$ : $<<0.05$; **: $\mathrm{p}<0.01$; and $* * *: \mathrm{p}<0.001$, Bonferroni post hoc test following oneway ANOVA versus the $\mathrm{NC}$; normal control prior diabetes was induced and before HOT: $p<0.05$; \#\#: $\mathrm{p}<0.01$; and \#\#\#: $\mathrm{p}<0.001$, Bonferroni post hoc test following one-way ANOVA versus Diab; diabetic (after diabetes induced and prior HOT), and ), and vs hyperlipidemic rabbits (after HL being induced and prior HOT), 4th, 10th treatment, and A10W; fourth treatment, tenth treatment, and 10 weeks after last treatment respectively.

\section{Liver function test}

In the diabetic and HL rabbit model induced by alloxan, after one week AST, ALT, LDH, and ALP levels were significantly increased compared to normal rabbits as shown in figure $5 \mathrm{a}$ and 5 b. However, after HOT treatments sessions were started these values showed significantly time-dependent reduction when compared to those before HOT was applied.
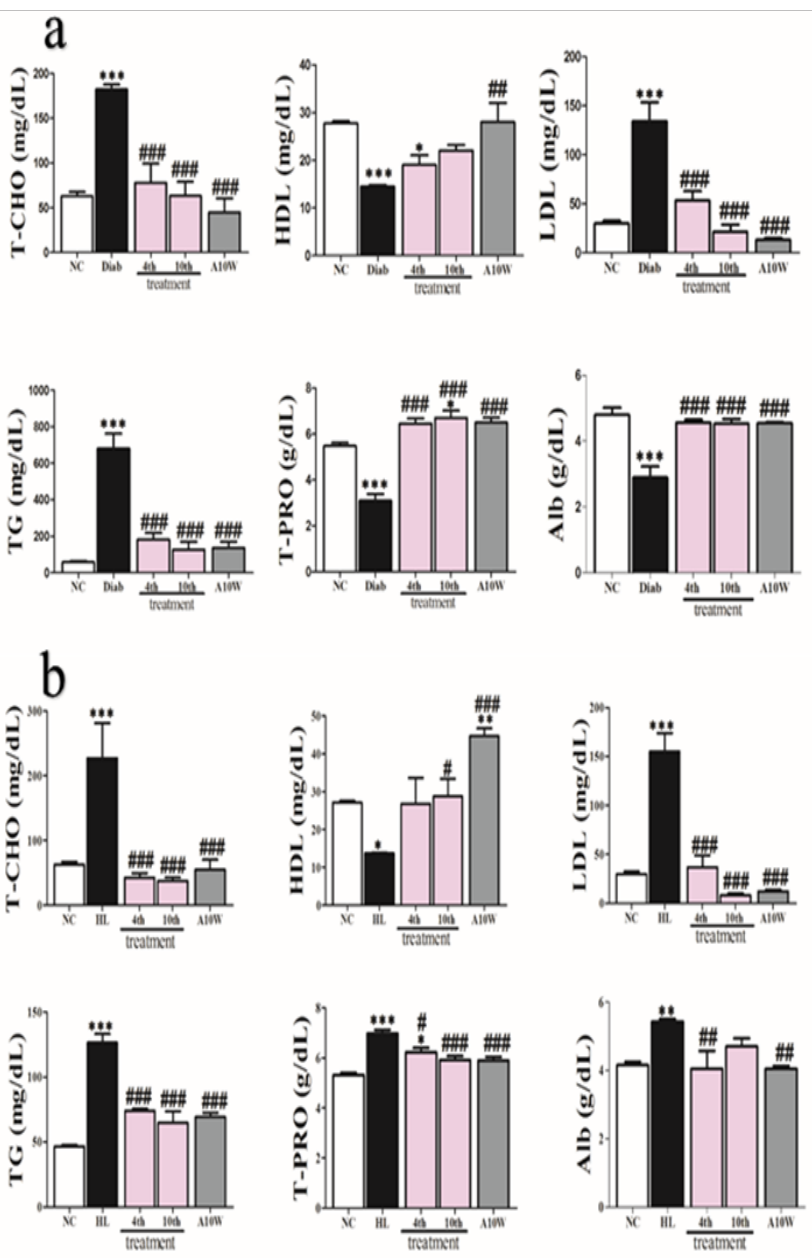

Figure 5: Effects of HOT on serum lipid and protein profiles in alloxan-induced diabetes (a) and poloxamar 407-induced hyperlipidemic rabbits (b). T-CHO, total cholesterol; HDL, high density lipoprotein; LDL, low density lipoprotein; TG, triglyceride; T-PRO, total protein; Alb, Albumin. Data were reported as means \pm SEMs $(\mathrm{n}=10)$. *: $\mathrm{p}<$ $0.05 ; * *: \mathrm{p}<0.01$; and ${ }^{* *}: \mathrm{p}<0.001$, Bonferroni post hoc test following oneway ANOVA versus the NC; normal control prior diabetes was induced and before HOT: $\mathrm{p}<0.05$; \#\#: $\mathrm{p}<0.01$; and \#\#: $\mathrm{p}<0.001$, Bonferroni post hoc test following one-way ANOVA versus Diab; diabetic (after diabetes induced and prior HOT), and vs hyperlipidemic rabbits (after HL being induced and prior HOT), 4th, 10th treatment, and $\mathrm{A} 10 \mathrm{~W}$; fourth treatment, tenth treatment, and 10 weeks after last treatment respectively.

\section{Lipid profile}

In the diabetic and HL rabbit model, T-CHO, LDL, TG, T-PRO, and Alb levels were increased significantly compared to those of normal rabbits as shown in figure $6 \mathrm{a}$ and $6 \mathrm{~b}$. However, after HOT treatments, these levels significantly decreased when compared to those before HOT were applied. In addition, HDL level significantly decreased compared to normal group. However, in rabbit subjected HOT treatment, the level was elevated significantly.

\section{Blood gas, Hb, Hct, and lactate}

Blood concentration of $\mathrm{PO}_{2}, \mathrm{SO}_{2}, \mathrm{Hb}$, and Hct were significantly decreased in diabetic rabbits compared to normal rabbits. However, in HOT-treated rabbits, these parameters significantly improved. Oppositely, $\mathrm{PCO}_{2}$ and lactate were significantly elevated in diabetic rabbits compared to normal rabbits. Never the less, 
in after HOT treatment, the concentration significantly reduced as shown in table 1.

Table 1: Effects of HOT on the blood gas, Hb, Hct, and lactate in alloxan induced-diabetes rabbits

Data were reported as means \pm SEMs $(\mathrm{n}=10) . *$ : $\mathrm{p}<0.05 ; * * \mathrm{p}<0.01$; and $* * *: \mathrm{p}<0.001$, Bonferroni post hoc test

\begin{tabular}{|l|l|l|l|l|l|}
\hline & NC & Diab & 4th & 10 th & A10W \\
\hline $\mathrm{pCO}_{2}(\mathrm{mmHg})$ & $21.59 \pm$ & $69.75 \pm$ & $31.10 \pm$ & $39.08 \pm$ & $36.04 \pm$ \\
& 1.09 & $16.60^{* * *}$ & $0.98^{\# \#}$ & $2.32^{\#}$ & $2.43^{\# \#}$ \\
\hline $\mathrm{pO}_{2}(\mathrm{mmHg})$ & $110.4 \pm$ & $47.0 \pm$ & $155.7 \pm$ & $100.5 \pm$ & $82.7 \pm$ \\
& 7.8 & $1.4^{* *}$ & $23.3^{\# \# \#}$ & $2.4^{\#}$ & 7.6 \\
\hline $\mathrm{SO}_{2}(\%)$ & $95.74 \pm$ & $72.45 \pm$ & $96.60 \pm$ & $87.13 \pm$ & $86.18 \pm$ \\
& 0.44 & $3.80^{* * *}$ & $1.80^{\# \#}$ & 4.76 & 4.93 \\
\hline $\mathrm{Hb}(\mathrm{g} / \mathrm{dL})$ & $12.26 \pm$ & $9.0 \pm$ & $12.48 \pm$ & $11.83 \pm$ & $13.93 \pm$ \\
& 0.27 & $0.35^{* * *}$ & $0.44^{\# \# \#}$ & $0.88^{\# \#}$ & $0.22^{*}, \# \#$ \\
\hline $\mathrm{Hct}(\mathrm{g} / \mathrm{dL})$ & $36.67 \pm$ & $31.75 \pm$ & $37.25 \pm$ & $35.33 \pm$ & $38.25 \pm$ \\
& 0.91 & 0.75 & 1.43 & 2.72 & 2.51 \\
\hline $\mathrm{Lac}(\mathrm{mM} / \mathrm{L})$ & $3.8 \pm$ & $9.7 \pm \pm$ & $5.46 \pm$ & $6.43 \pm$ & $7.0 \quad \pm$ \\
& 0.4 & $1.5^{* * *}$ & $0.6^{* * * \# \#}$ & 0.1 & $0.4^{*}$ \\
\hline
\end{tabular}

following one-way ANOVA versus the NC; normal control: $p<0.05$; $\# \#$ : $\mathrm{p}<0.01$; and \#\#\#: $<<0.001$, Bonferroni post hoc test following oneway ANOVA versus Diab; diabetic (after diabetes induced and prior HOT), 4th, 10th treatment, and A10W; fourth treatment, tenth treatment, and 10 weeks after last treatment respectively.

\section{Blood ions}

Blood concentration of $\mathrm{Mg}^{2+}$ and $\mathrm{Ca}^{2+}$ were significantly decreased in diabetic rabbits compared to normal rabbits. However, in HOT- treated rabbits, these parameters significantly increased. On the other side, $\mathrm{K}^{+}, \mathrm{Cl}^{-}$, and $\mathrm{AG}$ were significantly elevated in diabetic rabbits compared to normal rabbits. Never the less, in after HOT treatment, the concentration significantly reduced. $\mathrm{Na}^{+}$concentration although significantly reducedin diabetic rabbits compared to normal group did not increased after HOT treatment as shown in table 2.

Table 2: Effects of HOT on the blood electrolytic balance in alloxan induced-diabetes rabbits

\begin{tabular}{|l|l|l|l|l|ll|}
\hline & NC & Diab & 4 th & 10 th & A10W & \\
\hline $\mathrm{Na}^{+}(\mathrm{mmol} / \mathrm{dL})$ & 141.4 & 140.8 & 133.6 & $138.0 \pm$ & 139.2 & \pm \\
& \pm 0.6 & $\pm 0.4^{*}$ & \pm 2.5 & 61.3 & 0.8 & \\
\hline $\mathrm{Cl}^{-}(\mathrm{mmol} / \mathrm{dL})$ & 97.83 & 106.0 & 97.50 & $96.80 \pm$ & 99.36 & \pm \\
& \pm 0.70 & $\pm 0.24^{*}$ & $\pm 3.81^{\#}$ & $1.41^{\#}$ & $0.77^{\#}$ & \\
\hline $\mathrm{Mg}^{2+}(\mathrm{mmol} / \mathrm{dL})$ & $0.55 \pm$ & $0.43 \pm$ & $0.59 \pm$ & $0.56 \pm$ & 0.63 & \pm \\
& 0.00 & $0.03^{* * *}$ & $0.01^{\# \#}$ & $0.00^{\# \#}$ & $0.00^{* * * \# \#}$ \\
\hline $\mathrm{Ca}^{2+}(\mathrm{mmol} / \mathrm{dL})$ & $1.54 \pm$ & $1.37 \pm$ & $1.57 \pm$ & $1.49 \pm$ & 1.56 & \pm \\
& 0.009 & $0.031^{*}$ & $0.043^{\#}$ & 0.060 & $0.029^{\# \#}$ & \\
\hline $\mathrm{K}^{+}(\mathrm{mmol} / \mathrm{dL})$ & $4.53 \pm$ & $5.83 \pm$ & $4.89 \pm$ & $4.37 \pm$ & 4.50 & \pm \\
& 0.13 & $0.33^{*}$ & 0.22 & 0.64 & $0.19^{\#}$ & \\
\hline $\mathrm{AG}(\mathrm{mmol} / \mathrm{dL})$ & 14.51 & 20.90 & 14.05 & $14.67 \pm$ & 21.83 & \pm \\
& \pm 0.55 & $\pm 0.40^{*}$ & $\pm 1.40^{\#}$ & 0.88 & $2.93^{* *}$ & \\
\hline
\end{tabular}

Data were reported as means \pm SEMs $(n=10) . *: p<0.05 ; * *: p<0.01$ and $* * *: p<0.001$, Bonferroni post hoc test following one-way ANOVA versus the NC; normal control prior diabetes was induced and before HOT: $\mathrm{p}<0.05$; \#\#: $\mathrm{p}<0.01$; and \#\#\#: $\mathrm{p}<0.001$, Bonferroni post hoc test following one-way ANOVA versus Diab; diabetic (after diabetes induced and prior HOT), 4th, 10th treatment, and A10W; fourth treatment, tenth treatment, and 10 weeks after last treatment respectively.

\section{Discussion}

To best of our knowledge, there was no published data concerning effect of HOT in HL and diabetic animal models. However, in a previous study, the result indicated that UBI was safe when used in diabetic rabbits induced by alloxan. The glucose level was significantly reduced and the body weight was significantly increased after UBI treatments compared to diabetic rabbit prior UBI sessions were started ${ }^{[11]}$. This experiment followed the same protocol, however, the difference was only that the blood was perfused with oxygen for 10 seconds using sterile oxygen cylinder. A report stated that HOT treatment improves insulin efficiency and blood glucose levels were reduced. In addition, vision was improved in some diabetic patients with different stages of retinitis undergone HOT treatments because RBC aggregation was suppressed ${ }^{[12]}$.

Normally, protein metabolized and hence excreted by kidney as a urea which was considered a final product of protein and amino acid. High blood urea level indicates renal problem. BUN directly affected by protein intake and considered as an indicator of renal function ${ }^{[13]}$. The result in Figure 4 showed an increased level of Cre, BUN, UA, and CK, which in an agreement with other study which showed that an increased level of Cre, BUN, and UA in diabetes was well documented by ${ }^{[14]}$. In contrast, after treatment with HOT rabbits exhibited a significant reduction in the levels of these indicators, suggesting that HOT effectively enhanced renal function. As kidney was also responsible of regulating ions and electrolyte homeostasis, so an imbalance in electrolytes such as $\mathrm{Mg}^{2+}, \mathrm{Ca}^{2+}, \mathrm{K}^{+}, \mathrm{Na}^{+}, \mathrm{Cl}^{-}$considered a deterioration in kidney function of diabetic rats ${ }^{[15]}$. As shown in table 1 these indicators were significantly improved by HOT treatments.

AST, ALT, LDH, and ALP are indicators of liver function and their elevations indicated liver stress as mentioned ${ }^{[15,16]}$. These indicators as shown in Figure 5 were significantly reduced by HOT treatments which supports that HOT has hepatoprotective effect. This was in a good agreement with our previous study ${ }^{[11]}$ we reported that AST, ALT, LDH, and ALP were significantly improved after UBI treatment.

Our result in Figure 6 as expected showed an elevation in T-CHO, TG, LDL, and decreased level in HDL in diabetic rabbits before HOT sessions were started which agreed with a previous study ${ }^{[7]}$ who found that diabetic patients are under the risk of Hypertriglyceridemia and hypercholesterolemia with respect to heart and coronary disease. On other hand after HOT treatments, these indicators were significantly improved. Hence, HOT could be used to prevent cardiovascular disease. The result was in a good agreement with our previous study. T-PRO and Alb were significantly decreased in diabetic rabbits. However, after HOT treatment, these indicators were significantly increased, this result coincided with a conclusion made other investigators ${ }^{[11]}$.

Table 1 indicated that $\mathrm{pO}_{2}$ was significantly reduced in diabetic rabbits without $\mathrm{HOT}$ treatment whereas $\mathrm{pCO}_{2}$ was significantly increased in diabetic rabbits with HOT. However, these values were significantly improved after HOT treatments which agreed ${ }^{[17,18]}$. Lowered blood $\mathrm{pH}$, reduced $\mathrm{HCO}_{3}-$, and increased lactate levels were observed in the diabetic indicating acidic blood, which may be a sign of ketoacidosis ${ }^{[19]}$. 
This result coincided with Mattman and $\mathrm{Lida}^{[20]}$ who found that UBI improved oxygen delivery, blood elements, and stimulation of mitochondrial oxidation may help quick recovery of many aliments.

Alloxan causes a high reduction in insulin release by the destruction of the $\beta$-cells of the islets of Langerhans, results in hyperglycemia ${ }^{[21]}$. Impaired glucose tolerance was attained due to lack of insulin in alloxan-induced diabetic rats by destructing the $\beta$-cells which leads to type I diabetes ${ }^{[22]}$. However, insulin level in rabbits subjected to HOT were showed a significant increment in insulin level compared to diabetic rabbits. Fasting serum HbAlc level was an important indicator for patients with diabetes who have cardiovascular diseases. The HbAlc level has been found to be directly proportional to the blood glucose concentration $^{[14,23]}$. As expected, the HbA1c levels were increased in the diabetic groups in our study, which were lowered by the 10 times treatment protocol of HOT. This outcome could be due to improved insulin secretion ${ }^{[14]}$.

AST, ALT, LDH, and ALP which indicates liver injury caused by hyperglycemia andhyperlipidemia due to tissue insult $^{[24,25]}$. However, HOT enhanced the liver condition of hyperlipidemic rabbits. This result come in a good agreement with our previous study concluded that AST, ALT, ALP, and LDH significantly reduced in diabetic rabbits undergoing UBI for eight times with blood oxygenation ${ }^{[11]}$.

Dyslipidemia associated with chronic kidney diseases as concluded by Tsimihodimos $\mathrm{V}$ et al. ${ }^{[26]}$ However, after HOT was applied, hyperlipidemic rabbits showed a significant enhancement in kidney status which can be clarified by a significant decrement in the measured levels of parameters. These results coordinated with a recent study ${ }^{[11]}$ which found that in diabetic rabbits subjected to UBI treatment, a marked enhancement in kidney function namely CRE, BUN, and UA levels were lowered significantly. In our study the reason behind this could be attributed to UV implied in HOT which enhanced tissue blood perfusion and oxygenation.

\section{Conclusion}

The study evaluated the effectiveness of HOT in treatment of type 1 diabetes and HL by using auto-transfusion. Evaluation was performed through some biochemical and hematological parameters. As a result, HOT effectively lowered glucose level, improved lipid profile enhanced body weight in diabetic rabbits, showed a hepato-protective, cardio-protective and protects against renal damage. In addition to, blood gas and electrolytes imbalance were improved.

However, further study to elucidate the adverse effect of HOT and to elaborate in mechanism of action using some molecular and biological techniques. This experiment was a part of a series of experiments in our lab using to remind the scientific community about an old, cheap and effective method of treatment that forgotten for long time due to an advancement in synthetic and chemical pharmaceutical agents.

Acknowledgements: This research was supported by Basic Science Research Program through the National Research Foundation of Korea (NRF) funded by the Ministry of Education, Science, and Technology (2015R1D1A3A01019245).
Competing Interests: The authors declare that they have no competing interest.

\section{References}

1. $\mathrm{Wu}, \mathrm{X}, \mathrm{Hu}, \mathrm{X}$. , Hamblin, M.R. Ultraviolet blood irradiation: Is it time to remember "the cure that time forgot"? (2016) J Photochem Photobiol B 157: 89-96.

Pubmed | Crossref | Others

2. Wasan, K.M., Subramanian, R., Kwong, M., et al. Poloxamer 407-mediated alterations in the activities of enzymes regulating lipid metabolism in rats. (2003) J Pharm Pharm Sci 6(2): 189-197.

Pubmed | Crossref | Others

3. Johnston, T.P. The P-407-induced murine model of dose-controlled hyperlipidemia and atherosclerosis: a review of findings to date. (2004) J Cardiovasc Pharmacol 43(4): 595-606.

Pubmed | Crossref | Others

4. Waite, M., Martin, C., Franklin, R., et al. Human Factors and Data Logging Processes with the Use of Advanced Technology for Adults with Type 1 Diabetes: Systematic Integrative Review. (2018) JMIR Hum Factors 5(1): e11. Pubmed | Crossref | Others

5. Negrato, C.A., Tarzia, O. Buccal alterations in diabetes mellitus. (2010) Diabetol Metab Syndr 2:3.

Pubmed | Crossref | Others

6. Mendenhall, E., Kohrt, B.A., Norris, S.A., et al. Non-communicable disease syndemics: poverty, depression, and diabetes among low-income populations. (2017) Lancet 389(10072): 951-963.

Pubmed | Crossref | Others

7. Schwarz, P.E. Report from the Congress of the American Diabetes Association (ADA): Orlando 2005 - 65th Annual Scientific Sessions in San Diego, CA, USA, June 10th-14th 2005. (2005) Exp Clin Endocrinol Diabetes 113(8): 475479.

Pubmed | Crossref | Others

8. Kim, S.M., Lee, J.S., Lee, J., et al. Prevalence of diabetes and impaired fasting glucose in Korea: Korean National Health and Nutrition Survey 2001. (2006) Diabetes Care 29(2): 226-231.

Pubmed | Crossref | Others

9. Ramachandran, A., Snehalatha, C., Shetty, A.S., et al. Trends in prevalence of diabetes in Asian countries. (2012) World J Diabetes 3(6): 110-117.

Pubmed | Crossref | Others

10. Sorli, C. New developments in insulin therapy for type 2 diabetes. (2014) Am J Med 127(10 Suppl): S39-S48.

Pubmed | Crossref | Others

11. Kim G-B. Effects of Ultraviolet Blood Irradiation in a Diabetes Rabbit Model. (2016) J Diab Obes 3(3): 1-11.

Pubmed | Crossref $\mid$ Others

12. Gerich, J.E. Role of the kidney in normal glucose homeostasis and in the hyperglycaemia of diabetes mellitus: therapeutic implications. (2010) Diabet Med 27(2): 136-142. Pubmed | Crossref | Others

13. Lopez-Giacoman, S., Madero, M. Biomarkers in chronic kidney disease, from kidney function to kidney damage. 
(2015) World J Nephrol 4(1): 57-73.

Pubmed | Crossref | Others

14. Ikewuchi, C.C., Ikewuchi, J.C., Ifeanacho, M.O. Restoration of plasma markers of liver and kidney functions/ integrity in alloxan-induced diabetic rabbits by aqueous extract of Pleurotus tuberregium sclerotia. (2017) Biomed Pharmacother 95: 1809-1814.

Pubmed | Crossref | Others

15. Go, H.K., Rahman, M.M., Kim, G.B., et al. Antidiabetic Effects of Yam (Dioscorea batatas) and Its Active Constituent, Allantoin, in a Rat Model of Streptozotocin-Induced Diabetes. (2015) Nutrients 7(10): 8532-8544.

Pubmed | Crossref | Others

16. Giannini, E.G., Testa, R., Savarino, V. Liver enzyme alteration: a guide for clinicians. (2005) CMAJ 172(3): 367-379. Pubmed | Crossref | Others

17. Cao, Z., Cooper, M.E. Pathogenesis of diabetic nephropathy. (2011) J Diabetes Investig 2(4): 243-247. Pubmed | Crossref | Others

18. Gnudi, L., Thomas, S.M., Viberti, G. Mechanical forces in diabetic kidney disease: a trigger for impaired glucose metabolism. (2007) J Am Soc Nephrol 18(8): 2226-2232.

Pubmed | Crossref | Others

19. Cox, K., Cocchi, M.N., Salciccioli, J.D., et al. Prevalence and significance of lactic acidosis in diabetic ketoacidosis. (2012) J Crit Care 27(2): 132-137.

Pubmed | Crossref | Others

20. Mattman, L.H. Media Mas MM. (2001) In Cell wall deficient forms: Stealth Pathogens. 2nd ed: CRC press: 1. Pubmed $\mid$ Crossref $\mid$ Others

21. Yashwant Kumar, A., Nandakumar, K., Handral, M., et al. Hypoglycaemic and anti-diabetic activity of stem bark extracts Erythrina indica in normal and alloxan-induced diabetic rats. (2011) Saudi Pharm J 19(1): 35-42.

Pubmed | Crossref | Others

22. Tang, L.Q., Wei, W., Chen, L.M., et al. Effects of berberine on diabetes induced by alloxan and a high-fat/high-choles- terol diet in rats. (2006) J Ethnopharmacol 108(1): 109-115. Pubmed | Crossref | Others

23. Selvin, E., Steffes, M.W., Zhu, H., et al. Glycated hemoglobin, diabetes, and cardiovascular risk in nondiabetic adults. (2010) N Engl J Med 362(9): 800-811.

Pubmed | Crossref | Others

24. Korolenko, T.A., Johnston, T.P., Tuzikov, F.V., et al. Early-stage atherosclerosis in poloxamer 407-induced hyperlipidemic mice: pathological features and changes in the lipid composition of serum lipoprotein fractions and subfractions. (2016) Lipids Health Dis 15: 16.

Pubmed | Crossref | Others

25. Jiang, Z.G., Mukamal, K., Tapper, E., et al. Low LDL-C and high HDL-C levels are associated with elevated serum transaminases amongst adults in the United States: a cross-sectional study. (2014) PLoS One 9(1): e85366.

Pubmed $\mid$ Crossref $\mid$ Others

26. Tsimihodimos, V., Mitrogianni, Z., Elisaf, M. Dyslipidemia associated with chronic kidney disease. (2011) Open Cardiovasc Med J 5: 41-48.

Pubmed | Crossref | Others
Submit your manuscript to Ommega Publishers and we will help you at every step:

- We accept pre-submission inquiries

- Our selector tool helps you to find the most relevant journal

- We provide round the clock customer support

- Convenient online submission

- Thorough peer review

- Inclusion in all major indexing services

- Maximum visibility for your research

Submit your manuscript at

https://www.ommegaonline.org/submit-manuscript 